\title{
UM CASO DE APROPRIAÇÃO ATIVA DA PESQUISA ACADÊMICA QUE VEM SE FORMATANDO NO INTERIOR DO MATO GROSSO
}

\author{
A CASE OF ACTIVE APPROPRIATION OF ACADEMIC RESEARCH THAT HAS BEEN \\ FORMATTING IN THE INTERIOR OF MATO GROSSO
}

UN CASO DE APROPIACIÓN ACTIVA DE INVESTIGACIÓN ACADÉMICA QUE SE HA FORMATADO EN EL INTERIOR DE MATO GROSSO

\section{Erick dos Santos Silva \\ iD 9}

Doutorando em Ensino de Ciências pela Universidade de São Paulo (USP)

Professor EBTT do Instituto Federal do Rio de Janeiro (IFRJ)

Pós-Graduação em Ensino de Ciências da Natureza e Matemática do IFMT/JNA

ssf.erick@gmail.com

\section{Alexandre Alberto Visentin Ramos de Araujo (iD)}

Mestre em Ensino de Ciências pelo Instituto Federal do Rio de Janeiro (IFRJ)

Professor do Colégio Militar de Juiz de Fora (CMJF)

alexandrevisentin@gmail.com

\begin{abstract}
Resumo
O trabalho apresenta os resultados de uma investigação envolvendo os interesses de pesquisa de um grupo de pósgraduandos que também atuam como professores na rede básica de ensino. A partir de uma análise de conteúdo do material explorado, foi possível prospectar os principais temas pesquisados pelo grupo e observar que eles apresentam relação direta com a melhoria dos espaços pedagógicos e contextos acadêmicos que os cercam. As enunciações destes profissionais revelam que a busca por capacitação e conhecimentos pedagógicos compartilhados apresentam forte conexão com seus contextos e necessidades cotidianas. Os resultados apontam um caminho fértil para o trânsito dos resultados de pesquisa recentes até o chão de sala, em uma perspectiva de apropriação ativa realizada pelos pós-graduandos envolvidos.
\end{abstract}

Palavras-chave: Ensino de Ciências. Formação de Professores. Demandas educacionais contemporâneas.

Recebido em: 17 de abril de 2021.

Aprovado em: 18 de agosto de 2021.

Como citar esse artigo (ABNT):

SILVA, Erick dos Santos; ARAUJO, Alexandre Alberto Visentin Ramos de. Um caso de apropriação ativa da pesquisa acadêmica que vem se formatando no interior do Mato Grosso.

Revista Prática Docente, v. 6, n. 2, e052, 2021.

http://doi.org/10.23926/RPD.2021.v6.n2.e052.id1125 


\section{Abstract}

The work presents the results of an investigation involving the research interests of a group of graduate students who also act as teachers in the basic education network. From a content analysis of the collected material, it was possible to prospect the main themes researched by the group and observe that they have a direct relationship with the improvement of the pedagogical spaces and academic contexts that surround them. The statements of these professionals reveal that the search for training and shared pedagogical knowledge has a strong connection with their contexts and everyday needs. The results point to a fertile path for the transit of recent research results to the classroom floor, in a perspective of active appropriation carried out by the graduate students involved.

Keywords: Science Teaching. Teacher Education. Contemporary educational demands.

\section{Resumen}

El trabajo presenta los resultados de una investigación que involucra los intereses investigativos de un grupo de estudiantes de posgrado que también actúan como docentes en la red de educación básica. A partir de un análisis de contenido del material explorado, fue posible prospectar los principales temas investigados por el grupo y observar que tienen una relación directa con la mejora de los espacios pedagógicos y contextos académicos que los rodean. Las declaraciones de estos profesionales revelan que la búsqueda de formación y conocimiento pedagógico compartido tiene una fuerte conexión con sus contextos y necesidades cotidianas. Los resultados apuntan a un camino fértil para el tránsito de los resultados de las investigaciones recientes al piso del aula, en una perspectiva de apropiación activa realizada por los estudiantes de posgrado involucrados.

Palabras clave: Enseñanza de las ciencias. Formación de profesores. Demandas educativas contemporáneas. 


\section{INTRODUÇÃO}

A comunidade acadêmica, frequentemente, expõe sua preocupação a respeito da avaliação de impactos positivos, oriundos dos resultados de pesquisa, em contextos práticos ligados à sala de aula. A definição de critérios que permitam avaliar a utilidade e impacto das ideias investigadas no âmbito da pesquisa em ensino é tema recorrente em trabalhos publicados por importantes periódicos brasileiros (STUDART, 2001; PENA, 2004; SILVA, 2019; SILVA; SASSERON, 2020). No entanto, apesar de avanços observados na discussão da temática, aplicações efetivas dos resultados de pesquisa, em salas de aula, ainda parecem incipientes (PENA, 2004; MARANDINO, 2003; MORTIMER, 1996; SILVA, 2019; 2020). Em relação a isso, Marandino (2003) e Mortimer (1996) já destacavam a existência de resistências para o avanço dos resultados de pesquisa em direção às salas de aula. De acordo com os autores, tais resistências seriam ligadas a uma prática docente, no ensino de ciências, marcada por perspectivas tradicionais, condicionada a diferentes contextos políticos e econômicos ou, ainda, determinada por problemas originados durante a formação acadêmica dos profissionais educadores. Por exemplo, Mortimer (1996) aponta notável dificuldade na preparação de profissionais para atuação em contextos mais construtivistas, o que acaba nos levando a uma relação custo-benefício desfavorável: podemos investir bastante tempo na abordagem de poucos conceitos científicos e, muitas vezes, não obter ganhos mensuráveis em relação ao contexto tradicional vigente. Esse cenário ainda é uma preocupação atual (SILVA; SASSERON, 2020; STROUPE et.al., 2019).

Um dos caminhos apontados pela literatura especializada consiste em identificar, de maneira consistente, os conhecimentos gerados por pesquisas em ensino de ciências, considerando a complexidade atrelada ao fato desta pesquisa ter dimensões sociais e, assim, parte de seus estudos pode ser considerado pesquisa de base, sem uma obrigatória aplicabilidade imediata (STUDART, 2001; DELIZOICOV, 2004; PENA, 2004; STROUPE et.al., 2019). Por outro lado, a difusão dos resultados das pesquisas e os debates a seu respeito, em cursos de formação inicial e continuada, permitem ampliar o reconhecimento de boas práticas educacionais, ajudando a testá-las e validá-las em diferentes cenários práticos. Esse procedimento pode dar voz e incluir, na pesquisa acadêmica, os diferentes atores de sala de aula, que trabalham nos mais variados contextos educacionais (SILVA; SASSERON, 2020; SILVA, 2019). 
Nessa perspectiva, é possível vislumbrar a formação continuada como uma ponte para o trânsito de ideias educacionais entre pesquisa e sala de aula. Veremos um estudo de caso no interior do estado de Mato Grosso, onde a formação continuada tem se mostrado um ambiente propício para a difusão dos resultados de pesquisa em ensino e de reflexão sobre boas práticas educacionais, em um contexto de apropriação ativa. A partir da análise de conteúdo do material explorado (BARDIN, 1977; 2016; SILVA, 2020), foi possível categorizar os interesses e tendências de pesquisa de um conjunto de pós-graduandos e suas possíveis conexões com os contextos profissionais em que estão inseridos, conforme detalharemos na sequência.

\section{Proposta inVestigativa E AMOSTRA INVESTIGADA}

\subsection{Proposta inVestigativa e hiPótese de trabalho}

O trabalho investigou a viabilidade de um Programa de Pós-Graduação em Ensino de Ciências da Natureza e Matemática, no interior de Mato Grosso, proporcionar um caminho célere e fértil para que os resultados de pesquisa cheguem às salas de aula da região. Isso foi realizado a partir de uma pesquisa estruturada em torno da seguinte pergunta: Como estudantes da Pós-Graduação em Ensino de Ciências da Natureza e Matemática do Instituto Federal de Mato Grosso (PECNM-IFMT) articulam os resultados de pesquisa recentes com suas práticas pedagógicas?

A pergunta de pesquisa formatada assume, como hipótese de trabalho, que parte dos estudantes da PECNM-IFMT trabalha como professor, no ensino básico, em escolas do interior mato-grossense. Outra hipótese admitida é que o programa PECNM-IFMT coloca o estudante em contato com resultados de pesquisas recentes. Partindo dessas duas ideias básicas, estruturamos uma investigação para elencar os temas pesquisados pelos estudantes participantes, em conjunto com algumas de suas ideias sobre o ensino de ciências, tais como potencialidades e fraquezas. Os dados foram coletados a partir de registros escritos e quatro encontros gravados.

A partir de uma análise de conteúdo dos relatos da turma (BARDIN, 1977; 2016; SILVA, 2020) e informações complementares oriundas de quatro encontros remotos gravados, foi possível elaborar um agrupamento em classes dos principais temas de pesquisa desses estudantes. De acordo com Bardin (2016), podemos extrair ideias contidas nos diversos discursos cotidianos a partir de uma investigação técnica estruturada em quatro principais etapas: pré-análise, codificação, categorização e inferência (BARDIN, 1977; 2016; SILVA, 
2020). Em nosso contexto, os registros permitiram, ainda, a realização de um mapeamento das motivações práticas associadas a esses discursos. Analisando todo este material coletado, percebemos que vários estudantes atuam em diferentes contextos da educação básica, pesquisando sobre prática pedagógica ou metodologias de ensino-aprendizagem. Além disso, eles pretendem continuar, durante sua atuação profissional, com a investigação em ensino e, ainda, implementar possíveis melhorias oriundas dos resultados dessas pesquisas.

\subsection{GRUPO INVESTIGADO}

A presente pesquisa contou com a participação de um grupo de colaboradores que estavam na fase final de formação do Programa Lato Sensu de Pós-Graduação em Ensino de Ciências da Natureza e Matemática do Instituto Federal de Educação do Mato Grosso (PECNM-IFMT). O levantamento dos dados foi realizado a partir das atividades entregues pelos estudantes - questionários e textos discursivos - e também de enunciações complementares obtidas em quatro encontros remotos gravados.

Do total de treze estudantes matriculados na disciplina FÍSICA NO ENSINO DE CIÊNCIAS, dez conseguiram participar do formato remoto e, desse modo, contribuir para o levantamento de dados relativos ao programa de Pós-Graduação do IFMT (PECNM-IFMT).

\section{TEMAS DE PESQUISA, MOTIVAÇõES E ATUAÇÃO PROFISSIONAL DO GRUPO INVESTIGADO}

\subsection{INTERESSES DE PESQUISA DA TURMA INVESTIGADA}

As investigações foram realizadas considerando, como dados principais, relatos digitados pelos pós-graduandos. Os encontros gravados foram considerados como dados complementares e, portanto, qualquer análise referente a eles será explicitada ao longo do texto.

A análise de conteúdo que foi implementada revelou um possível agrupamento das respostas dadas pelos estudantes em duas categorias predominantes: uma associada a melhorias do professor ligadas à formação inicial e continuada; outra ligada aos aspectos tecnológicos da educação - como aulas remotas e tecnologias para o suporte e manutenção do ensino. Essas foram as duas principais enunciações dos pesquisados (BARDIN, 1977; 2016, p.217). A figura 1 sintetiza essas ideias. É relevante destacar que, entre a diversidade de temas possíveis no âmbito do PECNM-IFMT, percebe-se uma predominância de temas ligados à formação de professores.

Figura 1: 
Figura 1 - Distribuição percentual dos temas de pesquisa na categorização admitida

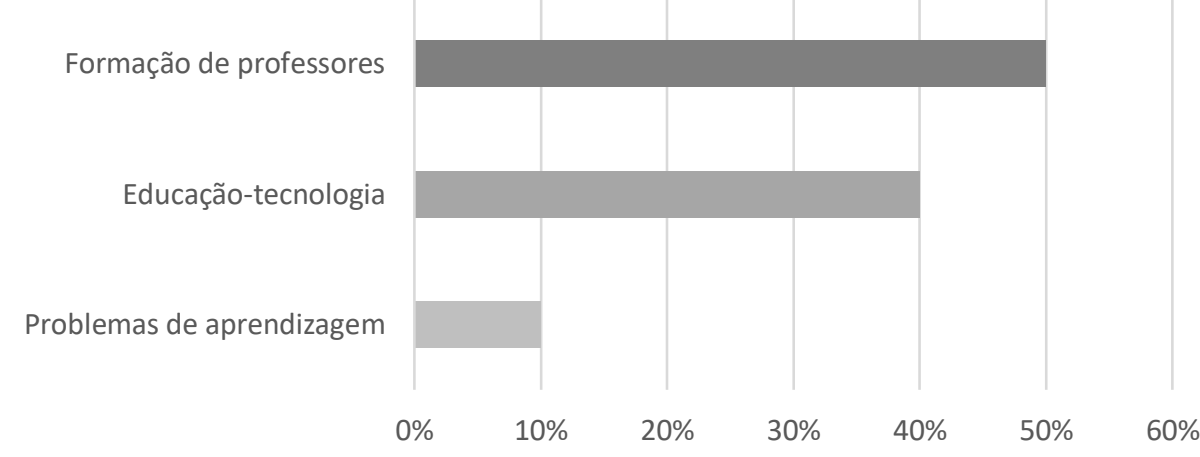

Fonte: Elaborada pelos autores em pesquisa sistemática realizada no âmbito do PECNM-IFMT.

\subsection{MOTIVAÇÕES DE PESQUiSA DA TURMA INVESTIGADA}

$\mathrm{Na}$ etapa de pré-análise, verificamos a possibilidade de categorizar outras duas enunciações da amostra pesquisada. Ao mencionarem os temas de pesquisa e apresentarem justificativas para tal escolha, os estudantes acabaram enunciando potencialidades e fraquezas do ensino de ciências atual, observados a partir de seus olhares e de seus respectivos ambientes de trabalho.

Em relação à motivação principal, foi relatada uma busca de melhoria do processo ensino-aprendizagem no qual estes estudantes estão inseridos. É possível identificar interesses particulares - como mudança salarial ou mudança de carreira - mas também notamos pontos de convergência entre alguns relatos. No decorrer da pesquisa, ao passar pelas etapas de Préanálise (I) e Codificação (II), percebemos a possibilidade de caracterizar um conjunto de potencialidades e fraquezas típicas presentes nas respostas fornecidas pelos estudantes sobre o ensino de ciências. Na etapa de Categorização (III), avançamos nessa caracterização secundária sobre potencialidades e dificuldades observadas no ensino de ciências.

Seguindo esse caminho, verificamos que $50 \%$ do grupo destacam como positiva a implementação de projetos educacionais de formação e capacitação, elogiando os recursos humanos envolvidos ou mobilizados nessas ações. Esses seriam as potencialidades do sistema educacional associadas ao ensino de ciências. O grupo destacou ainda a criatividade dos profissionais da educação na resolução de problemas - como a improvisação de espaços para práticas investigativas - e ressaltou que o ensino tecnológico se apresenta como uma boa alternativa para a inserção de jovens no mundo do trabalho (Fig. 2). Esses relatos apresentaram três enunciados principais, que podem ser agrupados em três categorias para as potencialidades: Programas de formação e capacitação, Soluções criativas e Ensino tecnológico. 
Figura 2 - Distribuição percentual das potencialidades atribuídas ao ensino de ciências

Programas de formação e capacitação

Soluções criativas

Ensino tecnológico

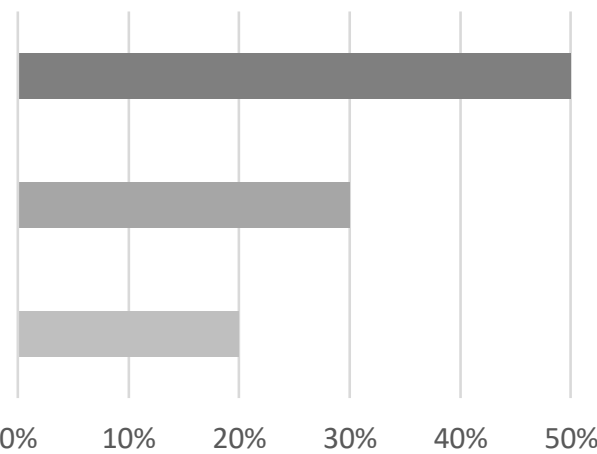

$50 \%$

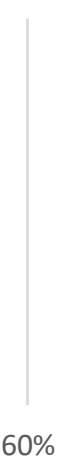

$60 \%$

Fonte: Elaborada pelos autores em pesquisa sistemática realizada no âmbito do PECNM-IFMT.

Em relação às principais dificuldades enfrentadas no ensino de ciências, $50 \%$ do grupo destacou a falta de infraestrutura e também a dificuldade de uma implementação mais efetiva de boas práticas científicas, associando isso a problemas de gestão dos recursos para o ensino de ciências - esses problemas foram vinculados, de maneira geral, aos governos municipal, estadual e federal. Os outros $40 \%$ destacaram problemas nos métodos e dificuldades de aprendizagem. Vale ressaltar, ainda, que $10 \%$ do grupo citou problemas específicos no processo ensino-aprendizagem (Fig. 3).

Figura 3 - Distribuição percentual de fraquezas atribuídas ao ensino de ciências

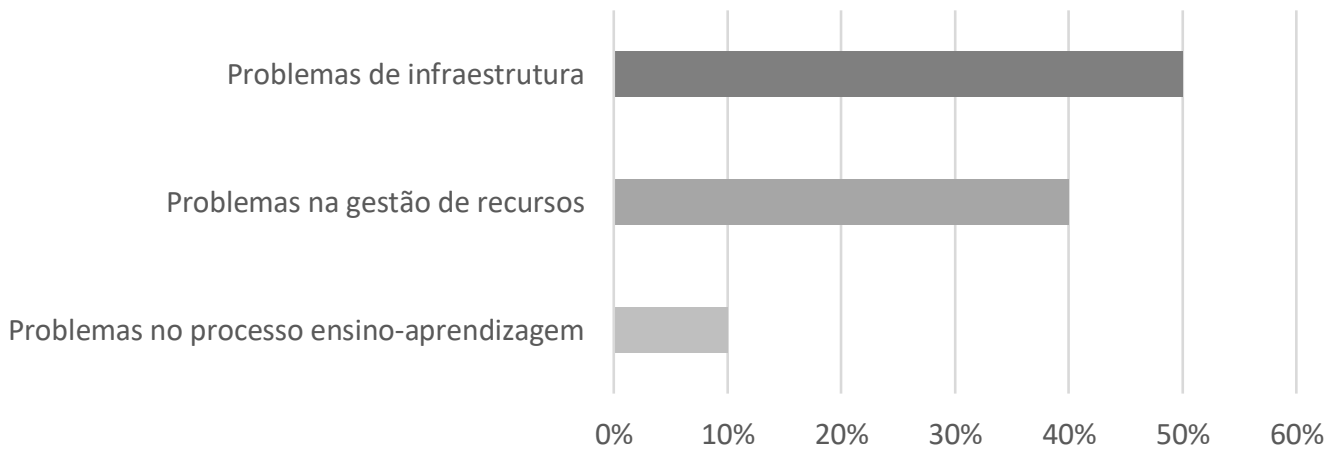

Fonte: Elaborada pelos autores em pesquisa sistemática realizada no âmbito do PECNM-IFMT.

\subsection{ATUAÇÃO PROFISSIONAL DA TURMA INVESTIGADA E PERSPECTIVAS FUTURAS}

Nos relatos escritos, foi possível identificar que 50\% dos estudantes trabalham no ensino básico. Na quarta e última etapa considerada em nossa análise de conteúdo, denominada Inferência, sentimos necessidade de confirmar essa informação e, para isso, lançamos mão de encontros gravados. Como algumas omissões foram percebidas nos relatos escritos, elaboramos perguntas sobre a atuação profissional para os encontros e confirmamos que, de fato, $50 \%$ dos pesquisados estão em atuação na educação básica (Fig. 4). Vale destacar que um pós-graduando 
atua como professor de Ciências e Matemática em uma aldeia indígena, no município de Juína, no estado de Mato Grosso.

Outro dado de interesse é que $90 \%$ do grupo investigado revela pretensão de seguir com a pesquisa em ensino, considerando a possibilidade de avançar até o doutorado. A figura 4 sintetiza essas informações.

Figura 4 - Estudantes que pretendem seguir atualmente com a pesquisa em ensino e avançar para um mestrado ou doutorado. Vale destacar que 50\% do grupo pesquisado está atuando no ensino básico

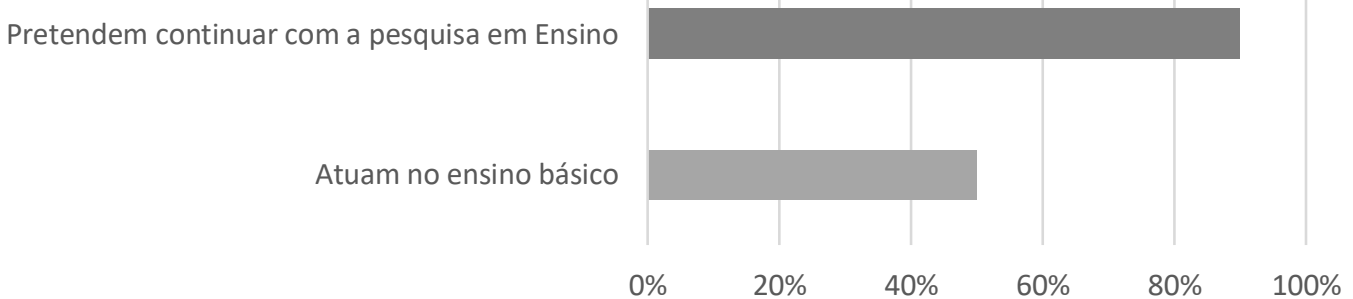

Fonte: Elaborada pelos autores em pesquisa sistemática realizada no âmbito do PECNM-IFMT.

\section{DiscuSSÃo}

Podemos retomar a pergunta de pesquisa norteadora do trabalho: Como estudantes da Pós-Graduação em Ensino de Ciências da Natureza e Matemática do Instituto Federal de Mato Grosso (PECNM-IFMT) articulam os resultados de pesquisa recentes com suas práticas pedagógicas?

É possível notar, a partir da análise de conteúdo implementada durante nossa pesquisa, consequências do contato dos pós-graduandos com os resultados de pesquisa recentes: seus interesses/temas de pesquisa incorporam resultados e discussões atuais no âmbito do ensino de ciências. Isso demonstra, então, a plausibilidade de nossa segunda hipótese de trabalho - onde admitimos que a PECNM-IFMT é um espaço de contato com ideias educacionais recentes. Contudo, voltando à primeira hipótese - onde admitimos a atuação dos estudantes no ensino básico - percebemos que ela se confirma parcialmente. A Fig.4 revela que 50\% da turma trabalha, atualmente, neste nível de ensino. No entanto, de forma complementar, podemos recorrer aos encontros gravados e corroborar que os outros $50 \%$ estão se dedicando à pósgraduação e revelam intenção de atuar na educação básica, mas no decorrer da pesquisa, não possuíam contratos profissionais formalizados - alguns deles estão à espera de aprovação em alguns concursos regionais específicos.

Uma vez verificada a plausibilidade geral das hipóteses de trabalho, podemos avançar para uma possível resposta da pergunta de pesquisa formata. Inicialmente, destacamos que a 
análise de conteúdo apontou uma necessidade dos estudantes em buscar capacitação, que pode ocorrer por meio de certificações ou por conhecimentos mobilizados em espaços de pesquisa. Além disso, a pesquisa demonstra que os estudantes pretendem realizar melhorias em seus ambientes de trabalho e práticas profissionais diretamente relacionadas com os resultados de pesquisas recentes. Ao pesquisarem por temas ligados principalmente à formação profissional e ao binômio educação-tecnologia, os estudantes revelam-se abertos para a busca de aperfeiçoamentos associados ao ensino de ciências, mostrando participação ativa neste processo.

Nesse contexto, como os estudantes do programa articulam os resultados de pesquisa com suas respectivas práticas pedagógicas? A análise desenvolvida ao longo deste trabalho aponta para uma articulação viva - e calcada na prática - entre os conhecimentos mobilizados pela pesquisa acadêmica e a atuação profissional dos pós-graduandos. Seus trabalhos e discursos revelam inquietações da prática pedagógica cotidiana, o que confere tangibilidade ao processo no qual esses professores/pesquisadores buscam conhecimentos teórico-práticos para melhorar as relações de ensino-aprendizagem promovidas, nos diferentes contextos profissionais e acadêmicos onde estão inseridos. Trata-se, assim, de uma apropriação ativa, onde inquietações do chão de sala entram numa interlocução com os resultados de pesquisa mais gerais apresentados pela literatura.

\section{CONSIDERAÇõES FinAIS}

Este trabalho permite apontar alguns interesses de pesquisa recentes prospectados em um espaço de pós-graduação no interior do Mato Grosso, juntamente com algumas de suas motivações práticas. Podemos perceber que o trânsito de ideias, em mão dupla, pode ajudar na formatação de pesquisas mais alinhadas às demandas reais, oriundas do chão de sala. A análise de conteúdo implementada revelou um forte interesse dos pós-graduandos em questões ligadas não só à formação profissional, mas também ao binômio educação-tecnologia. Isso está diretamente conectado com as demandas educacionais e discussões mais recentes observadas no âmbito do ensino de ciências (STROUPE et.al., 2019, THOMPSON et al., 2019; SILVA; SASSERON, 2020).

Por fim, além de contribuir para inserir a comunidade escolar (e suas questões) no debate acadêmico, o processo de melhoria contínua do acesso e manutenção de espaços de pósgraduação permitem uma desejada oxigenação de ideias e, quem sabe, de melhorias educacionais mais concretas. 


\section{REFERÊNCIAS}

BARDIN, Lawrence. Análise de conteúdo. $1^{\text {a }}$ ed. São Paulo: Edições 70, 2016 (Obra original publicada em 1977).

DELIZOICOV, Demétrio. Pesquisa em ensino de ciências como ciências humanas aplicadas. Caderno Brasileiro de Ensino de Física, v. 21, n. 2, p. 145-175, 2004.

https://periodicos.ufsc.br/index.php/fisica/article/view/6430

MARANDINO, Martha. A prática de ensino nas licenciaturas e a pesquisa em ensino de ciências: Questões atuais. Caderno Brasileiro de Ensino de Física, v. 20, n. 2, p. 168-193, 2003. https://periodicos.ufsc.br/index.php/fisica/article/view/6544

MORTIMER, Eduardo Fleury. Construtivismo, mudança conceitual e Ensino de ciências: para onde vamos? Investigações em Ensino de Ciências, v. 1, n. 1, p. 20-39, 1996. https://www.if.ufrgs.br/cref/ojs/index.php/ienci/article/view/645/436

PENA, Fábio Luís Alves. Por que, apesar do grande avanço da pesquisa acadêmica sobre ensino de Física no Brasil, ainda há pouca aplicação dos resultados em sala de aula? Revista Brasileira de Ensino de Física, v. 26, n. 4, p. 293-295, 2004.

https://www.scielo.br/pdf/rbef/v26n4/a02v26n4.pdf

SILVA, Erick dos Santos. Concepções de Licenciandos sobre a Relação EnsinoAprendizagem e a Existência de uma Barreira Tradicional a ser Transposta. Educação Matemática em Revista, v. 25, n. 66, p. 114-136, 2020.

http://sbem.iuri0094.hospedagemdesites.ws/revista/index.php/emr/article/view/1824/pdf

SILVA, Erick dos Santos. ENEM, prática docente e metodologias ativas: uma equação que não fecha. Caderno Brasileiro de Ensino de Física, v.36, n.1, p.55-68, 2019.

https://periodicos.ufsc.br/index.php/fisica/article/view/2175-7941.2019v36n1p55

SILVA, Erick dos Santos; SASSERON, Lúcia Helena. BNCC: entre avanços de pesquisa e articulações necessárias. Currículo e Docência, v.2, n.2, p.23-45, 2020.

https://periodicos.ufpe.br/revistas/CD/article/view/249558

STROUPE, David, MOON, Jean; MICHAELS, Sarah. Introduction to special issue: Epistemic tools in Science education. Science Education, v. 103, n. 4, p. 948-951, 2019. https://onlinelibrary.wiley.com/doi/abs/10.1002/sce.21512

STUDART, Nelson. Uso da Pesquisa em Ensino de Física na prática docente. Revista Brasileira de Ensino de Física, v. 23, n. 3, p.259, 2001. https://www.scielo.br/pdf/rbef/v23n3/v23n3a01.pdf

THOMPSON, Jéssica, HAGENAH, Sara, MCDONALD, Scott; BARCHENGER, Christie. Toward a practice-based theory for how professional learning communities engage in the improvement of tools and practices fors cientific modeling. Science Education, v. 103, n. 6, p. 1423-1455, 2019. https://onlinelibrary.wiley.com/doi/abs/10.1002/sce.21547 